NASA Contractor Report 4251

\title{
Spatial Three-Dimensional Secondary Instability of Compressible Boundary-Layer Flows
}

Nabil M. El-Hady

Analytical Services and Materials, Inc. Hampton, Virginia

Prepared for

Langley Research Center

under Contract NAS1-18599

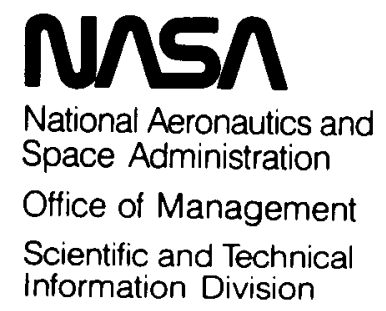

1989 


\title{
SPATIAL THREE-DIMENSIONAL SECONDARY INSTABILITY OF COMPRESSIBLE BOUNDARY-LAYER FLOWS
}

Nabil M. El-Hady †

Analytical Services and Materials, Inc., Hampton, Virginia 23666

\begin{abstract}
Three-dimensional linear secondary instability theory is extended for compressible and high Mach number boundary-layer flows. The effect of small but finite amplitude compressible Tollmien-Schlichting wave on the growth of three-dimensional perturbations is investigated. The focus is on principal parametric reson ance responsible for the strong growth of subharmonics in low disturbance environment. The effect of increasing Mach number on the onset, growth, shape of eigenfunctions of the subharmonics is assessed, and the resulting vortical structure is examined.
\end{abstract}

July 21,1989

† Senior Rese arch Scientist 


\section{SPATIAL THREE-DIMENSIONAL SECONDARY INSTABILITY OF COMPRESSIBLE BOUNDARY-LAYER FLOWS}

Nabil M. El-Hady $\dagger$

Analytical Services and Materials, Inc., Ham pton, Virginia 23666

\section{INTRODUCTION}

Transition to turbulence is a complex phenomenon that has recieved considerable attention in the last decade. Our knowledge of the process by which laminar incompressible flows undergoes transition and reaches a turbulent state has increased exponentially in recent years. On the other hand, high speed counterpart research in this area is severely lagging behind. Until very recently, renewed interest in high speed flight prompted more activities in transition research for compressible and high Mach number fiows.

While instability and transition in comperessible flows is our main concern in this paper, the relevance of the incompressible stability theory to transition mechanisms will be briefly reviewed. The road to transition in Blasius-type flow involves several stages. First, an initial stage of linear instability of small disturbances, called primary instability, is well described by the Orr-Summerfeld equation. This is followed by a second stage where apparant nonlinearity is revealed by the amplification of three dimensionality and the unusually high growth rate of these disturbances. At this stage, the disturbances are still weak and the nonlinear distortion to the mean flow is negligable. Finally, a strong and shorter nonlinear stage takes place with high and intense fluctuations that leads to the inevitable fully turbulent regime.

† Senior Rese arch Scientist 
Recent progress in the early nonlinear stage where strong three dimensionality takes place has identified a major link in the transition process between the linear and fully nonlinear stages. It showed that there is a well defined transition from laminar two-dimensional (2D) to laminar three-dimensional (3D) waves through a secondary instability mechanism. This mechanism has been harmonically clarified by numerous experiments [1-9], small disturbance theories [10-14], as well as by numerical simulations [15-20]. Exellent reviews on the subject of secondary instability mechanisms for incompressible flows has been provided by Herbert [21] and Bayly et al. [22].

Our current understanding of the 3D phenomenon leading to transition in incompressible boundary-layer flows indicates two major types of breakdown. In both, a 3D lambda-shaped vortex structure appears with streamwise as well as spanwise periodicity. In the first type, the lambda vortices are aligned in the streamwise direction with the same period as the primary wave and with a spanwise periodicity of the same order. This type is called fundamental breakdown or peak-valley splitting, and is refered to as K-type breakdown. The second type of breakdown is known by the appearance of staggered lambda vortices with a streamwise period twice that of the primary wave. This is called subharmonic breakdown. Incompressible experiments indicate that the subharmonic breakdown occurs when the amplitude of the primary wave is low or moderate, while the fundamental type occurs for high primary amplitudes. However, numerical simulations show that the appearace of one type of breakdown or the other depends largely on the spectrum of the background disturbances $[23,24]$.

For incompressible flows, Herbert $[13,14]$ formulated a linear secondary in stability theory for $3 D$ waves in the presence of a finite amplitude $2 D$ wave. His theory can predict the increasingly $3 \mathrm{D}$ behavior with large growth rates that occur in both the fundamental and subharmonic types of breakdown. Craik [10] established a resonant triad model that comprised of a $2 \mathrm{D}$ wave and two oblique waves which can predict an instability of the subharmonic type. Craik's mechanism, known as C-type, is though to 
dominate at low amplitude of the primary 2D wave, while Herbert's subharmonic mechanism, known as $\mathrm{H}$-type, reflects the situation at moderate amplitude of the primary wave. For 3D incompressible boundary layers on swept wings, El-Hady [25] established a resonant triad model that comprised of different 3D instability modes, that may include stationary crossflow, traveling crossflow, vertical vorticity, and Tollmien-Schlishting modes. El-Hady's model can predict a large amplification of a superharmonic or a subharmonic wave depending on the initial spectrum of the amplitude and phases of the triad components.

In contrast to this progress for incompressible flows, only the initial stage of compressible linear stability is well developed for high speed flows. The link between linear regime and observed transition at high speeds is yet to be identified. What makes it a difficult task is that experimental knowledge of stability features in the linear as well as nonlinear ranges are extremely poor for compressible flows and do not allow a physical input to model transition mechanisms. However, incompressible mechanisms of secondary instability can serve as a guide until we have better feedback from clean compressible stability experiments.

For compressible and high-speed boundary-layer flows, the stability problem is more complex. Here, it is necessary to account for the rate of heat transfer between the fluid and the surface. A thermal boundary layer with mean density variations develops in addition to the velocity boundary layer and it plays an important role in the determination of the stability of the flow. Compressibility is known to have a stabilizing effect on the primary wave due to a change in the nature of the instability as mach number increases. For incompressible and low Mach number flows, the instability is dominated by viscosity, while it is inviscidely dominated at high Mach numbers. A comprehensive review of the linear compressible stability theory of boundary layers has been provided by Mack [26]. We mention here some general results of the theory that are of particular importance to the transition process. In contrast to incompressible 
flows, where a 2D disturbance $\left(\psi=0^{\circ}\right)$ is mostly unstable, an oblique disturbance (called first mode) with $\psi$ of about $40^{\circ}-60^{\circ}$ is the most unstable at low Mach numbers. As Mach number increases, multiple eigenvalues of amplified and damped modes result as solution to the compressible stability equations. The leading unstable mode (called second mode) and higher ones are most unstable as 2D waves.

High speed stability experiments have been carried out on flat plates [27-29], and cones [30-32], but they were directly concerned with the first (linear) stage of transition. Some of these experiments were more successful than other in verifying the compressible stability theory, but none was designed to study the breakdown of the laminar boundary layer or the structure of the flow field near transition. The need is certainly urgent for this type of stability experiments to identify the link between the linear stage and observed transition at high speed. Recently, Erlebacker and Hussaini [33], by using a direct simulation of parallel compressible boundary layer at Mach 4.5, generated numerically a vortical structure qualitatively similar to the K-type breakdown for incompressible flows. They observed a secondary instability triggered by the interaction between a finite amplitude 2D wave with a 3D (first mode) disturbance. This instability at Mach 4.5 was found to be weaker than those found in incompressible flow.

In this paper, we extend the linear secondary three-dimensional instability theory and apply it to compressible and high speed flows. We investigate the effect of small but finite amplitude 2D or oblique compressible TS wave on the growth of 3D perturbations in compressible boundary layers. The focus here is on the growth of secondary subharmonics due to its importance in low disturbance environment. We study how the subharmonic instability mechanism will sustain as Mach number increases. Some early results of this work were described by the author elsewher [34]. In section 2 the analysis is developed. Section 3 discusses the numerical procedures. Results and discussions are given in section 4 . Then we end with concluding remarks. 


\section{ANALYSIS}

The flow field is described by the laminar compressible 3D Navier-Stokes and energy equations. Lengths, velocities, and time are made dimensionless using a reference length $L=\left(v_{\infty} x / U_{\infty}\right)^{1 / 2}$, the freestream velocity $U_{\infty}$ and $L / U_{\infty}$ respectively. Here $x$ is the distance from the leading edge of the flat plate and $v_{\infty}$ is the kinematic viscosity coefficient evaluated at the freestream. The pressure is made dimensionless using $\rho_{\infty} U_{\infty}^{2}$. The temperature, density, specific heats, viscosity, and thermal conductivity of air (treated as perfect gas) are made dimensionless using their corresponding freestream values. In terms of these dimensionless quantities and in a vectorial form, the governing equations read

$$
\begin{gathered}
\frac{\partial \rho}{\partial t}+\nabla \cdot(\rho \mathbf{V})=0 \\
\frac{\partial(\rho \mathbf{V})}{\partial t}+\nabla \cdot(\rho \mathbf{V V})=-\nabla p+\frac{1}{R} \nabla \cdot \tau \\
\rho\left(\frac{\partial \theta}{\partial t}+\mathbf{V} \cdot \nabla \theta\right)=(\gamma-1) M_{\infty}^{2}\left[\frac{\partial p}{\partial t}+\mathbf{V} \cdot \nabla p+\frac{1}{R} \mathbf{\Phi}\right]+\frac{1}{R P_{r}} \nabla \cdot(\mu \nabla \theta)
\end{gathered}
$$

with the state equation

$$
\gamma M_{\infty}^{2} p=\rho \theta
$$

In the above equations $\tau$ is the dimensionless viscous stress tensor, and $\Phi$ is the dimensionless dissipation function. They are defined as

$$
\begin{gathered}
\tau=\mu\left[\nabla \mathbf{V}+(\nabla \mathbf{V})^{T}\right]+\lambda \nabla . \mathbf{V I} \\
\boldsymbol{\Phi}=\tau: \nabla \mathbf{V}
\end{gathered}
$$

Also, $\gamma$ is the ratio of specific heats, $M_{\infty}$ is the freestream Mach number, $R=\rho_{\infty} U_{\infty} L / \mu_{\infty}$ is Reynolds number, $P_{r}=c_{p} \mu / k$ is Prandtl number, $\mu$ and $\lambda$ are the first and second coeffients of viscosity respectively, $I$ is a unit tensor, and $T$ denotes a transpose. 


\subsection{The Basic Flow}

We consider a basic flow which is assumed to be a solution of the equations of motion. This flow consists of a $2 \mathrm{D}$ compressible locally parallel boundary layer modulated by a small but finite amplitude compressible TS or oblique wave, here called primary disturbance. The basic flow takes the form

$$
\hat{q}(x, y, t)=q_{0}(y)+A\left[q_{1}(y) e^{i\left(\alpha_{r} x-\omega x\right)}+c c .\right]+O\left(A^{2}\right)
$$

where

$$
A \equiv A(x)=A_{0} e^{-\int \alpha_{i} d x}
$$

and cc denotes complex conjugate. We shall consider the variation of the primary amplitude $A(x)$ in Eq (8) to be weak. This variation will be neglected, and $A$ is assumed to be locally constant. Also, we shall neglect tems $O\left(A^{2}\right)$ in the analysis, and assume that the $2 \mathrm{D}$ compressible flow is modulated only by a periodic component of the linear stability problem. Justification of these assumptions will be considered later.

In Eq (7), $q_{0}$ stands for boundary-layer flow quantities $u_{0}, p_{0}, \theta_{0}, \rho_{0}$, and $\mu_{0}$, while $q_{1}$ stands for the eigensolutions of the primary disturbance, they are $u_{1}, v_{1}, p_{1}, \theta_{1}, \rho_{1}$, and $\mu_{1}$. These quantities represent velocities, pressure, temperature, density, and viscosity respectively. The eigensolutions are normalized such that the amplitude $A$ of the primary disturbance measure directly the maximum root mean square value of the disturbance velocity in the flow direction, that is

$$
\max _{0 \leq} 2\left[u_{1}(y)\right]^{2}=1
$$

For a spatial stability analysis, $\alpha$ is taken as the complex wavenumber for the primary disturbance defined as $\alpha=\alpha_{r}+i \alpha_{i}$, and $\omega$ is the real frequency.

In the analysis, the $x$-axis is always identified with the direction of the primary wave vector and the $y$-axis is defined normal to it. With that in mind, a $2 \mathrm{D}$ or oblique TS wave used to modulate the $2 \mathrm{D}$ compressible boundary layer will always be 
considered a 2D primary wave with respect to the secondary instability. While our focus in this study is on $2 \mathrm{D}$ basic state, this setup is designed for a future investigation of 3D basic state.

The eigensolutions of the primary wave are governed by a six order-system of equations that are given in Ref. [35-37]. The primary density disturbance is related to the temperature and pressure disturbances through the state equation

$$
\gamma M_{\infty}^{2} p_{1}=\rho_{1} \theta_{0}+\rho_{0} \theta_{1}
$$

while the primary viscosity disturbance is assumed to be linearly related to the temperature disturbance as

$$
\mu_{1}=\frac{d \mu_{0}}{d \theta_{0}} \theta_{1}+O\left(A^{2}\right)
$$

\subsection{The Secondary Instability}

To study the linear 3D instability of the basic flow given by (7), we superpose a small unsteady disturbance on each velocity, thermodynamic and transport quantity of the basic flow, that is

$$
q(x, y, z, t)=\hat{q}(x, y, t)+q_{2}(x, y, z, t)
$$

where $q_{2}$ is a secondary disturbance quantity that stands for disturbance velocities $u_{2}, v_{2}, w_{2}$, pressure $p_{2}$, temperature $\theta_{2}$, density $\rho_{2}$, and viscosity $\mu_{2}$. In a linear analysis, the secondary disturbance $q_{2}$ is assumed small compared to the primary disturbance $q_{1}$, such that the primary will influence the modulation of the secondary but not vise versa.

The basic flow given by (7) should include, in principle, all Fourier components of the primary wave, leading to very complicated interactions. This probably will hide the essential features of the instability mechanism. By taking a single Fourier component in this analysis, we basically neglect the nonlinear distortion of the eigensolutions $q_{1}$ at 
finite amplitude of the primary wave. This has been justified in the incompressible secondary instability theory [21] on the basis that the 3D secondary instability occurs at small amplitudes of the primary wave where the nonlinear distortion is weak. It was also noticed that the vortical nature of the $3 \mathrm{D}$ secondary instability is not affected by the nonlinearity. These justifications are still valid for the compressible version of the secondary instability theory, if we accept the notion that compressibility will probably retard and attenuate any nonlinear distortion.

Equation (12) is substituted into Eqs.(1-6), the basic flow is subtracted, and the resulting equations are linearized with respect to the secondary disturbance $q_{2}$. We end up with five coupled partial differential equations for the secondary 3D instability. The coefficients of these stability equations are functions of the basic flow and its derivatives, they are independent of the coordinate $z$, and periodic in $x$ and $t$. Hence, the $z$ variation can be separated, and Floquet theory of differential equations with periodic coefficients can be applied to give a solution to these equations in the form

$$
q_{2}(x, y, z, t)=e^{\gamma x+\sigma t} e^{i \beta z} \tilde{\phi}(x, y, t)
$$

wher $\beta$ is a real spanwise wavenumber of the secondary disturbance. It is a measure of the angle of divergence of the secondary disturbance propagation direction from the primary wave vector. $\gamma=\gamma_{r}+i \gamma_{i}$ and $\sigma=\sigma_{r}+i \sigma_{i}$ are two complex characteristic exponents, and $\tilde{\phi}(x, y, t)$ is a periodic function of $(x-\omega t / \alpha)$, the same as the period of the basic flow. We express $\tilde{\phi}(x, y, t)$ in terms of Fourier series to obtain the following expression for $q_{2}(x, y, z, t)$

$$
q_{2}(x, y, z, t)=e^{\gamma x+\sigma r} e^{i \beta z} \sum_{n=-\infty}^{\infty} \phi_{n}(y) e^{i n \alpha(x-\omega t / \alpha)}
$$

with

$$
\phi_{-n}=\bar{\phi}_{n}
$$

where the overbar denotes complex conjugate. 
Equation (14) represent a general Floquet form for the eigenmodes of a periodic basic flow. The subharmonic and fundamental modes are special cases of this form. The solution of the resulting eigenvalue problem will determine only two of the four real exponents $\gamma_{r}, \gamma_{i}, \sigma_{r}$ and $\sigma_{i}$ in $\mathrm{Eq}(14)$. The other two values must be given. For the purpose of our study of the spatial instability of subharmonic modes, we let $\gamma$, represent the growth rate of the secondary disturbance, $\sigma_{r}=0$ ( no temporal growth), $\sigma_{i}=-\omega / 2$ for a pure subharmonic mode, and let $\gamma_{i}$ represent the shift in the streamwise wavenumber of the secondary wave with respect to the primary one.

In the numerical treatment, the lowest possible truncation of Fourier series for subharmonic modes is used ( $n \leq 1)$, and no attempt was made to study the effect of that on the solution. Using Eqs.(14) and (15), the governing equations for the 3D subharmonic instability can be written in the form

$$
\left(a D^{2}+b D+c\right) \phi=A\left(d D^{2}+e D+f\right) \bar{\phi}+A^{2}(g D+h) \phi+. .
$$

where

$$
\phi \equiv\left(u_{2}, v_{2}, w_{2}, \rho_{2}, \theta_{2}\right)^{T},
$$

$D=d / d y$, and $a, b, c, d, e, f, g$, and $h$ are $5 \times 5$ matrices that are dependent upon the basic flow. The nonzero elements of these matrices are given in Appendix A ( except that for $g$ and $h$ ).

In Eq.(16) the density secondary disturbance $\rho_{2}$ is substituted for the pressure secondary disturbance $p_{2}$ by $u$ sing the state equation

$$
p M{ }_{\infty}^{2} p_{2}=\left(\rho_{0}+\rho_{1}\right) \theta_{2}+\left(\theta_{0}+\theta_{1}\right) \rho_{2}
$$

while the temperature secondary disturbance $\theta_{2}$ is substituted for the viscosity secondary disturbance $\mu_{2}$ by using Taylor's expansion of the total viscosity to yield

$$
\mu_{2}=\left(\frac{d \mu_{0}}{d \theta_{0}}+\frac{d^{2} \mu_{0}}{d \theta_{0}^{2}} \theta_{1}+\frac{1}{2} \frac{d^{3} \mu_{0}}{d \theta_{0}^{3}} \theta_{1}^{2}\right) \theta_{2}+. .
$$


We recall that in the construction of the basic flow, the effect of the finite primary amplitude $A$ on the basic flow was neglected. On the other hand, its effect on the secondary instability was taken into account. Because of that, terms of $O\left(A^{2}\right)$ and higher might appear beside terms $O(A)$ as a forcing function at the right hand side of Eq.(16). These higher order terms are unique for the compressible secondary instability equations in contrast to that for incompressible flow where only terms $O(A)$ appear. They arise because instabilities due to density as well as viscosity are taken into consideration in the compressible stability analysis. One might argue that these terms should be neglected in consistency with the expansions (7) and (11), or because the whole analysis of the secondary instability is based on a small finite amplitude $A$. For that reason, an evaluation of the effect of terms $O\left(A^{2}\right)$ on the secondary instability is considered in section 4 for the range of the primary amplitudes and Mach numbers used in the calculations.

The system of Eqs.(16) govern the secondary 3D spatial subharmonic instability of compressible 2D flows. They are five coupled ordinary differential equations for $u_{2}, v_{2}, w_{2}, \rho_{2}$ and $\theta_{2}$. When supplemented with homogeneous boundary conditions, they constitute an eigenvalue problem

$$
\gamma=\Gamma(\alpha, \beta, R ; A)
$$

for a given boundary layer velocity and temperature profiles $u_{0}(y)$ and $\theta_{0}(y)$ respectively.

\section{NUMERICAL PROCEDURES}

The governing equations of the mean flow (Appendix B) are numerically integrated by using a combination of a shooting technique and Runge-Kutta and Adam-Moulton integrator. The thermodynamic and transport properties of the perfect gas are computed at each integration step, as they vary with the temperature according to the relations given in Appendix B. 
The primary instability which modulate the 2D compressible boundary layer is governed by six first-order set of ordinary differential equations. They are numerically integrated as initial value problem using a freestream solution as initial condition. The solution of this eigenvalue problem was calculated before by Mack [35] and El-Hady $[36,37]$.

With respect to the secondary instability, the system of Eqs.(16) may be written as eight first-order complex equations in the form

$$
\begin{aligned}
D Z_{1} & =Z_{2} \\
D Z_{2}+l_{21} D \bar{Z}_{2} & =c_{2}+l_{22} \\
D Z_{3}+l_{31} D \bar{Z}_{3} & =c_{3}+l_{32} \\
D Z_{4} & =Z_{5} \\
D Z_{5}+l_{51} D \bar{Z}_{5} & =c_{5}+l_{52} \\
D Z_{6}+l_{61} D \bar{Z}_{6} & =c_{6}+l_{62} \\
D Z_{7} & =Z_{8} \\
D Z_{8}+l_{81} D \bar{Z}_{8} & =c_{8}+l_{82}
\end{aligned}
$$

with the boundary conditions,

$$
\begin{array}{r}
Z_{1}=Z_{3}=Z_{4}=Z_{7}=0 \quad \text { at } y=0 \\
Z_{1}, Z_{3}, Z_{4}, Z_{7} \rightarrow 0 \quad \text { as } y \rightarrow \infty
\end{array}
$$

where

$$
\begin{aligned}
& Z_{1}=u_{2}, Z_{2}=D u_{2}, Z_{3}=v_{2}, Z_{4}=w_{2}, \\
& Z_{5}=D w_{2}, Z_{6}=\rho_{2}, Z_{7}=\theta_{2}, Z_{8}=D \theta_{2},
\end{aligned}
$$

and $l_{m 1}$, and $l_{m 2}, m=1,2, . .8$ are both functions of the primary amplitude $A$, whereas $c_{m}, m=1,2, . .8$ are functions of the secondary disturbance quantities $Z_{m}, m=1,2, . .8$. In case of no modulation of the mean flow by a primary instability $\left(l_{m 1}=l_{m 2}=0\right)$, then the 
reduced system (21) will govern the stability of a primary subharmonic 3D wave.

We assume that the amplitude of the primary vanishes far in the freestream at $y \geq y_{e}, e$ denotes the edge of the boundary layer. Then the system (21) will have constant coefficients, and can be solved analytically producing four linearly independent, exponentially decaying solutions to conform with the boundary condition (23). With the freestream solution as initial condition, Eqs (21) are integrated from $y=y_{e}$ to $y=0$ at the wall, using a variable step-size algorithm [38], based on the Runge-Kutta -Fehlburg fifth-order formulas. The solution is orthonormalized at a preselected set of points using a modified Gram-Schmidt procedure. A Newton-Raphson technique is used to iterate on the eigenvalue to satisfy the last wall boundary condition within a specified accuracy of $O\left(10^{-5}\right)$.

\section{RESULTS AND DISCUSSION}

Fundamental changes in the compressible mean flow as well as in the character of the primary wave is expected as Mach number increases. These changes in turn will influence the modulated basic flow under study and its stability characteristics. In the subsonic range, a primary wave with fixed frequecy will propagate faster downstream acquiring longer wavelength as Mach number increases, and less time will be allowed for the wave amplitude to grow for a given length. Although $2 \mathrm{D}$ primary waves are most unstable in this range of Mach numbers, 3D primary waves exist in a natural background, and might trigger a secondary instability of the basic flow similar to the case of the damped vertical vorticity modes in incompressible flows $[25,39,40]$. As Mach number increases, 3D primary waves become dominant. Nevertheless, we focus our study in this paper on the possibility of the formation of strong three dimensionality from purely compressible $2 \mathrm{D}$ basic flow. 


\subsection{Onset and Growth of Secondary Subharmonics}

For 2D primary waves, Fig.(1) shows a decrease in the growth rates as Mach number increases for a wave with nondimensional frequency $F=60$ defined as

$$
F=10^{6} \omega / R
$$

The figure shows that the first and the second neutral points as well as the streamwise locations where maximum growth rates occur shift to the left, they occur earlier upstream, as Mach number increases.

Almost in the same region where primary $2 \mathrm{D}$ waves are growing, primary 3D subharmonics with broad band of spanwise wavelengths are subject to amplification. An example is given in Fig.(2) where growth rate curves are shown for a primary 3D subharmonic $(F=30)$ at different Mach numbers and fixed spanwise wavelength. The growth rates of the primary $3 D$ subharmonics like that of the primary $2 D$ waves are so small to compare with the highly amplified three-dimensionality that likely to be observed near transition. These rates of growth are induced by viscous instability at this range of Mach numbers.

A parametrical exitation by the finite amplitude primary wave will produce strong growth of secondary 3D subharmonics along a broad band of spanwise wavelengths. Figure (3) shows an example of such strong growth rates at $M_{\infty}=0.8$, as function of the spanwise wavenumber parameter $B$ defined as

$$
B=10^{3} \beta / R
$$

Calculations were performed at a downstream location corresponding to $R=850$ (see Fig.1) for different amplitudes of the primary 2D wave. Figure (3) has basically the same features of the growth of subharmonics in incompressible flows, the large values of the growth rates, the broad band of unstable subharmonics, and the sharp cutoff at low spanwise wavenumbers. As the primary amplitude decreases, growth rates as well as unstable band of secondary subharmonics sharply decrease. The results given in 
Fig.(3) are for real eigenvalues $\left(\gamma_{r}>0\right.$ and $\left.\gamma_{i}=0\right)$ that prove to be the dominant secondary subharmonic mode at $M_{\infty}=0.8$. This mode is perfectly synchronized with the basic flow similar to the case of incompressible flows [38]. Some calculations for the complex eigenvalues are marked in Fig.(3) for comparison.

Figure (4) compares the growth rates of the dominant real mode at Mach numbers $0.4,0.8,1.2,1.6$, and 2.2. Calculations were performed at $R=850$ and for a primary 2D amplitude $A=.01$ ( $R=750$ for $M_{\infty}=1.6$ and 2.2, and $A=.015$ for $\left.M_{\infty}=2.2\right)$. At these conditions, Fig.(4) indicates that compressibility has a stabilizing effect on the secondary subharmonic instability with considerable decrease in the growth rates and reduction in the unstable band of spanwise wavenumbers. With the increase of Reynolds number and/or the primary wave amplitude, the growth rates increase for all Mach numbers at a fixed frequency $F$.

Apart from the fact that slower growth rates of the primary wave is expected as Mach number increases, the onset of the primary instability also shifts to an upstream location, see Fig (1). Because of that, the outcome of a local compressibility contest (at fixed $R$ ) might somtimes prove misleading for fixed primary wave amplitude [34]. An example of that effect is shown in Fig (5a) at $R=850$, where the growth rates of the dominant subharmonic mode (real mode) are compared at $M_{\infty}=0.4$ and $M_{\infty}=0.8$ for primary wave amplitudes $A=.002$ and $A=.01$. At $A=.01$ compressibility appears to have a stabilizing influence at all unstable range of spanwise wavenumbers. As the amplitude A decreases, this stabilizing effect of compressibility starts to diminish until it is reversed at low enough primary amplitude such that at $A=.002$ it will appear that compressibility has a destabilizing influence on the growth rates. Figure (5b) provides same conclusions but at higher Reynolds number, $R=1050$. Moreover, it also shows that local compressibility effect might depend not only on the value of the primary wave amplitude, but also on the value of the spanwise wavenumber. 
The overall effect of compressibility on the growth of the secondary subharmonics can better be evaluated when combining the influence of increasing the amplitude $A$ of the primary wave, and the influence of increasing $R$ at various Mach numbers and for fixed frequency $F$. Figure (6a) shows the variation of the growth rates of the secondary subharmonic with $R$ for different Mach numbers, and for a spanwise wavenumber parameter $B=.15$. This value was chosen to average the most unstable band of spanwise wavenumbers in the range of Mach numbers used in the investigation. In these calculations, an initial amplitude $A_{0}=.001$ was assumed for the primary wave for all Mach numbers (at the streamwise location where the onset of the primary instability sets in), and its growth was taken into account while marching downstream. Figure (6b) shows the variation of the amplification factors with $R$ of the subharmonics calculated from the streamwise location where the onset of the secondary subharmonic sets in. Figure (6b) shows the amplification factors decreasing from about 29 at $M_{\infty}=0$ to 12 at $M_{\infty}=.8$. Corresponding variations of the growth rates and the amplification factors for the primary wave at different Mach numbers are included in Figs (6a) and (6b) for comparison.

The strong growth of the secondary subharmonics compared to that for the primary waves (specially in the region of interest where they are mostly unstable) justifies the assumption of neglecting the variation of the primary amplitude and considering it as locally constant. While Figs.(6a) and (6b) show that the growth rates and amplification factors of the secondary subharmonics decrease with increasing Mach number, they also indicate that the onset of the secondary instability is almost not affected by that increase. This may be due to the effect of the upstream shift of the onset location of the primary instability combined with our assumption that the initial primary amplitude $A_{0}$ is the same at all Mach numbers. In a real experimental environment it is expected that $A_{0}$ be proportional to $M_{\infty}^{2}$, and this might offset the stabilizing effect of compressibility as Mach number increases. 


\subsection{Effect of Mach Number on The Eigenfunctions}

Figure (7a) shows the variation across the boundary layer of the normalized root meam square values of the secondary subharmonic disturbance at different Mach numbers. The corresponding variations for the primary disturbances are shown in Fig. (7b) for comparison. These calculations were performed for $F=60, A=.01, B=.15$, and $R=850\left(R=750\right.$ for $M_{\infty}=1.6$ and 2.2, $A_{0}=.015$ for $\left.M_{\infty}=2.2\right)$. As Mach number increases the critical layer moves away from the wall (its location is shown in Fig 7), and the location of the maximum amplitude of $u_{1}$ and $\theta_{1}$ primary fluctuations, and $u_{2}, \theta_{2}$, and $w_{2}$

secondary fluctuations follow. We notice that, first, the secondary $v_{2}$-fluctuation is very small compared to the $u_{2}$-fluctuation, while the primary $v_{1}$-fluctuation is about $30 \%$ of the $u_{1}$-fluctuation at all Mach numbers. Second, the secondary $\theta_{2}$-fluctuation does not become that large as expected with the increase in Mach number (about $.25 u_{2}$ at $M_{\infty}=1.2$ ) compared to the primary $\theta_{1}-$ fluctuation (about $.75 u_{1}$ at $M_{\infty}=1.2$ ). Third, the secondary $w_{2}$-fluctuation which has a considerable amplitude at low Mach numbers (about $.50 u_{2}$ at $M_{\infty}=0$.) diminishes as Mach number increases. This last observation might prove responsible for diminishing the spanwise velocity variations as Mach number increases, and consequently reducing the production of the streamwise vorticity.

All calculations in this section were performed with terms $O\left(A^{2}\right)$ in $\operatorname{Eq}(16)$ taken into account. Yet, an estimate of the effect of these terms on the growth of the real subharmonic mode is given in table 1 at different Mach numbers and spanwise wavenumber parameters. The table shows that terms $O\left(A^{2}\right)$ have negligable effect on $\gamma_{r}$ within the accuracy limits used in these calculations. 


\subsection{Effect of Mach Number on The Vortical Structure}

Figure (8) shows the initial 2D vorticity contours of the basic flow ( has only a spanwise component) in a wave-fixed coordinate system at different Mach numbers. The peak vorticity occures near the wall and extends to the critical layer with a slower rate as Mach number increases. The strength of the vorticity becomes higher with the increase of the amplitude of the primary wave.

With the onset of the secondary instability, three-dimensionality is induced and the initial 2D vorticity is deformed producing a vortical structure. Figure (9) shows the spanwise vorticity contours in the $x-y$ plane at $z=0$ for different Mach numbers. The figure was plotted over four primary wavelengthes. These vortices are inclined to the mean flow direction at an angle near $30^{\circ}$ at $M_{\infty}=.4$ and increases to near $45^{\circ}$ at $M_{\infty}=2.2$. As Mach number increases, these vortices extend to the critical layer with less tilting angle into the $x-z$ plane.

Figure (10) shows contours of the streamwise vorticity produced by the spanwise velocity variations. The figure is plotted over two spanwise wavelengthes in the $z-y$

plane at $x=0$, for different Mach numbers. It shows counter-rotating vortices extending away from the wall toward the critical layer as Mach number increases. The interaction between the streamwise vorticity and the deformed spanwise component is the main drive to flow breakdown.

\section{CONCLUDING REMARKS}

The theory of linear three-dimensional secondary instability is extended to compressible and high spead boundary-layer flows. The effect of small but finite amplitude two-dimensional compressible Tollmien-Schlichting wave on the growth of threedimensional subharmonics is investigated for a range of Mach numbers to 2.2.

Numerical results performed for a primary wave with frequency $F=60$ show that the local effect of compressibility on the growth of the secondary subharmonics at fixed 
$R$ can be misleading. This effect may appear stabilizing or destabilizing depending on the amplitude of the primary wave, as well as the spanwise wavelength of the secondary wave.

However, the overall effect of increasing Mach number (including the influence of increasing both $R$ and $A$ ) is a reduction in the growth rates and the amplification factors of the secondary subharmonics, when the initial amplitude of the primary wave is kept fixed at all Mach numbers.

The maximum of the root mean square values of the secondary subharmonic eigenfunctions move away from the wall, following the critical layer, as Mach number increases. This suggests that the exchange of energy among the secondary disturbance, the mean flow, and/or the primary wave takes place around this location at different Mach numbers.

At low Mach numbers, the secondary spanwise velocity perturbation has a considerable amplitude. This amplitude decreases with the increase in Mach number, which might affect the production of the streamwise vorticity resulting in slowing or delaying the flow breakdown. This point worth a forthcoming separate investigation.

\section{ACKNOWLEGEMENT}

This work was supported by NASA Langley Research Center, under contract NAS1 - 18599 .

$$
\begin{aligned}
& \text { APPENDIX A: Nonzero Elements of Matrices a, b, c, d, e, and } f \text { in Equ (16) } \\
& a_{11}=-R^{-1} \mu_{0}, \quad a_{22}==m_{2} R^{-1} \mu_{0}, \quad a_{33}=-R^{-1} \mu_{0}, \quad a_{55}=-\left(R P_{r}\right)^{-1} D \mu_{0} \\
& b_{11}=-R^{-1} D \mu_{0}, \quad b_{12}=-R^{-1} \mu_{0} m_{1}(\gamma+i \alpha / 2), \quad b_{15}=-R^{-1} \hat{\mu}_{0} D u_{0}, \quad b_{21}=b_{12}, \\
& b_{22}=-R^{-1} m_{2} D \mu_{0}, \quad b_{23}=-R^{-1} m_{1} \mu_{0} \beta, \quad b_{24}=\left(\gamma M_{\infty}^{2}\right)^{-1} \theta_{0}, \quad b_{25}=\left(\gamma M_{\infty}^{2}\right)^{-1} \rho_{0},
\end{aligned}
$$




$$
\begin{aligned}
& b_{32}=b_{23}, \quad b_{33}=-b_{11}, \quad b_{42}=\rho_{0}, \quad b_{51}=-2 R^{-1} \mu_{0}(\hat{\gamma}-1) M_{\infty}^{2} D u_{0}, \quad b_{55}=2 a_{55}, \\
& c_{11}=\rho_{0}\left[(\gamma+i \alpha / 2) u_{0}-i \omega / 2\right]-R^{-1} \mu\left[m_{2}(\gamma+i \alpha / 2)^{2}-\beta^{2}\right] \\
& c_{12}=\rho_{0} D u_{0}-R^{-1} D \mu_{0}(\gamma+i \alpha / 2), \quad c_{13}=-R^{-1} m_{1} \mu_{0} \beta(\gamma+i \alpha / 2), \\
& c_{14}=\left(\gamma M_{\infty}^{2} \rho_{0}\right)^{-1}(\gamma+i \alpha / 2), \quad c_{15}=\left(\gamma M_{\infty}^{2}\right)^{-1} \rho_{0}(\gamma+i \alpha / 2), \\
& d_{11}=-R^{-1} \mu_{0} \theta_{1}, \quad d_{22}=-R^{-1} m_{2} \mu_{0} \theta_{1}, \quad d_{33}=-R^{-1} \mu_{0} \theta_{1}, \quad d_{55}=-\left(R P_{r}\right)^{-1} \mu_{0} \theta_{1}, \\
& e_{11}=\rho_{0} v_{1}-R^{-1} D\left(\hat{\mu}_{0} \theta_{1}\right), \quad e_{12}=-R^{-1} \mu_{0}\left[m_{1}(\bar{\gamma}+i \alpha / 2)+\bar{\gamma}-i \alpha / 2\right] \theta_{1}, \\
& e_{21}=-R^{-1} \hat{\mu}_{0}\left[m_{1}(\bar{\gamma}-i \alpha / 2)+i \alpha\right] \theta_{1}, \quad e_{22}=\rho_{0} v_{1}-R^{-1} m_{2} D\left(\hat{\mu}_{0} \theta_{1}\right), \\
& e_{23}=-R^{-1} m_{1} \hat{\mu}_{0} \theta_{1}, \quad e_{24}=\left(\gamma M_{\infty}^{2}\right)^{-1} \theta_{1}, \quad e_{25}=-R^{-1} \hat{\mu}_{0}\left(i m_{1} \alpha u_{1}+m_{2} D v_{1}\right)+\left(\hat{\gamma} M_{\infty}^{2}\right)^{-1} \rho_{1}, \\
& e_{32}=-e_{23} \beta, \quad e_{33}=e_{11}, \quad e_{42}=\rho_{1}, \quad e_{44}=v_{1}, \\
& e_{51}=2(\hat{\gamma}-1) M_{\infty}^{2} R^{-1}\left(i \alpha \mu_{0} v_{1}+\mu_{0} D u_{1}+\hat{\mu}_{0} \theta_{1} D u_{0}\right), \quad e_{52}=2(\hat{\gamma}-1) M_{\infty}^{2} R^{-1} \mu_{0}\left(i m_{1} \alpha u_{1}+m_{2} D v_{1}\right), \\
& e_{54}=-(\uparrow-1) \gamma^{-1} v_{1} \theta_{0}, \quad e_{55}=p^{-1} \rho_{0} v_{1}-\left(R P_{r}\right)^{-1}\left(2 \mu_{0} D \theta_{1}+\theta_{1} D \mu_{0}\right),
\end{aligned}
$$$$
f_{11}=\left[(\bar{\gamma}+i \alpha / 2) u_{0}+i \omega / 2\right] \rho_{1}+\rho_{0}(\bar{\gamma}+i \alpha / 2) u_{1}-R^{-1} \mathfrak{\mu}_{0}\left[m_{2}(\bar{\gamma}-i \alpha / 2)(\bar{\gamma}+i \alpha / 2)-\beta^{2}\right] \theta_{1},
$$$$
f_{12}=\rho_{1} D u_{0}+\rho_{0} D u_{1}-R^{-1}(\bar{\gamma}-i \alpha / 2) D\left(\hat{\mu}_{0} \theta_{1)}, \quad f_{13}=-R^{-1} \mu_{0} \beta\left[m_{1}(\bar{\gamma}-i \alpha / 2)+i m \alpha\right] \theta_{1},\right.
$$$$
f_{14}=i\left(\alpha u_{0}-\omega\right) u_{1}+v_{1} D u_{0}+\left(\hat{\gamma} M_{\infty}^{2}\right)^{-1}(\bar{\gamma}+i \alpha / 2) \theta_{1},
$$$$
f_{15}=R^{-1} \mathfrak{A}_{0}\left(m_{2} \alpha[i(\bar{\gamma}-i \alpha / 2)+\alpha] u_{1}+\left[i m_{1} \alpha+m(\bar{\gamma}+i \alpha / 2)\right] D v_{1}+D^{2} u_{1}\right\}
$$$$
+\left(\beta M_{\infty}^{2}\right)^{-1}(\bar{\gamma}+i \alpha / 2) \rho_{1}-R^{-1} \mu_{0} D\left(\theta_{1} D u_{0}\right)-R^{-1} \mu_{0} \theta_{1} D u_{0} D \theta_{0}-R^{-1}\left(D u_{1}+i \alpha v_{1}\right) D \mu_{0},
$$$$
f_{21}=i \alpha \rho_{0} v_{1}-R^{-1} m(\bar{\gamma}-i \alpha / 2) D\left(\beta_{0} \theta_{1}\right),
$$$$
f_{22}=\left[(\bar{\gamma}-i \alpha / 2) u_{0}+i \omega / 2\right]+\rho_{0}\left[(\bar{\gamma}-i \alpha / 2) u_{1}+D v_{1}\right]-R^{-1} \mathfrak{A}\left[(\bar{\gamma}-i \alpha / 2)^{2}+i \alpha(\bar{\gamma}-i \alpha / 2)-\beta^{2}\right] \theta_{1},
$$$$
f_{23}=-R^{-1} m \beta D\left(\mu_{0} \theta_{1}\right), \quad f_{24}=i\left(\alpha u_{0}-\omega\right) v_{1}+\left(\beta M_{\infty}^{2}\right)^{-1} D \theta_{1},
$$ 


$$
\begin{aligned}
& f_{25}=R^{-1} \hat{\mu}_{0}(i m \alpha+\bar{\gamma}+i \alpha / 2) D u_{1}+[i(\bar{\gamma}-i \alpha / 2)-\alpha] v_{1}+m_{2} D^{2} v_{1}-R^{-1} D \mu_{0}\left(i m \alpha u_{1}+m_{2} D v_{1}\right) \\
& -R^{-1} \mu_{0}(\bar{\gamma}+i \alpha / 2) \theta_{1} D u_{0} \\
& f_{31}=R^{-1} \hat{\mu}_{0} b r t a[(\bar{\gamma}+i \alpha / 2)+m(\bar{\gamma}-i \alpha / 2)] \theta_{1}, \quad f_{32}=R^{-1} \beta D\left(\hat{\mu}_{0} \theta_{1}\right), \\
& f_{33}=\left[(\bar{\gamma}-i \alpha / 2) u_{0}+i \omega / 2\right] \rho_{1}+\rho_{0}(\bar{\gamma}-i \alpha / 2) u_{1}-R^{-1} \hat{\mu}_{0}\left[(\bar{\gamma}-i \alpha / 2)^{2}+i \alpha(\bar{\gamma}-i \alpha / 2)-m_{2} \beta^{2}\right], \\
& f_{34}=-\left(\gamma M_{\infty}^{2}\right)^{-1} \beta \theta_{1}, \quad f_{35}=R^{-1} \hat{\mu}_{0} m \beta\left(i \alpha u_{1}+D v_{1}\right)-\left(\gamma M_{\infty}^{2}\right)^{-1} \beta \rho_{1}, \\
& f_{41}=(\bar{\gamma}+i \alpha / 2) \rho_{1}, \quad f_{42}=D \rho_{1}, \quad f_{43}=\beta \rho_{1}, \quad f_{44}=(\bar{\gamma}+i \alpha / 2) u_{1}+D v_{1}
\end{aligned}
$$

where $m=2(e-1) / 3$ is the ratio of the second viscosity coefficient $\lambda$ to the first viscosity coefficient $\mu, e=0$ corresponds to the Stokes hypothesis.e is taken 0.8 in this analysis. Also, $m_{1}=m+1, m_{2}=m+2, \mathfrak{\mu}_{0}=d \mu_{0} d d \theta_{0}, \mu_{0}=d \mathfrak{\mu}_{0} d d \theta_{0}$, and $\mu_{0}=d^{2} \mu_{0} d d \theta_{0}^{2}$.

\section{APPENDIX B: The Compressible Mean Flow}

The 2D compressible boundary-layer equations for zero pressure gradient can be derived using the transformation

$$
\eta=\frac{\left(R_{x}\right)^{1 / 2}}{x} \int_{0}^{y} \rho d y
$$

and a stream function to satisfy the continuity equation, where $R_{x}$ is the freestream $x-$ Reynolds number, $\rho$ is the density, $x$ is the distance along the plate and $y$ is the normal to it. In the similarity coordinate $\eta$, the governing equations are

$$
\begin{gathered}
\left(\rho \mu u^{\prime}\right)^{\prime}+g u^{\prime}=0 \\
\left(P_{r}^{-1} \rho \mu \theta^{\prime}\right)^{\prime}+g \theta^{\prime}+2 \rho \mu u^{\prime 2}=0
\end{gathered}
$$

where

$$
\begin{gathered}
2 g=\int_{0}^{\eta} u d \eta \\
\theta=\left(h-h_{e}\right) /\left(h_{o e}-h_{e}\right)
\end{gathered}
$$

and $h_{o}$ is the stagnation enthalpy. These equations are supplemented with the 
boundary conditions

$$
\begin{array}{r}
u=0, \theta^{\prime}=0, g=0 \quad \text { at } \eta=0 \\
u \rightarrow 1, \quad \theta \rightarrow \text { oas } \eta \rightarrow \eta_{e}
\end{array}
$$

The variation of of the viscosity $\mu$ and thermal conductivity $k$ of the perfect gas with temperature $\theta$ are given by

$$
\begin{aligned}
& \mu=\frac{1.458 \theta^{1.5} \times 10^{-5}}{\theta+110.4} \text { for } \theta>110.4 \\
& \mu=\left(0.693873 \times 10^{-6}\right) \theta \text { for } \theta \leq 110.4 \\
& k=\frac{0.6325 \theta^{0.5} \times 10^{-5}}{1+(245.4 / \theta) \times 10^{-12 / \theta}} \text { for } \theta>80 \\
& k=\left(0.222964 \times 10^{-6}\right) \theta \text { for } \theta \leq 80
\end{aligned}
$$

where $\mu$ is in $\mathrm{gm} / \mathrm{cm} \mathrm{sec}, k$ is in $\mathrm{cal} / \mathrm{cm} \mathrm{sec} C$, and $\theta$ is in $\mathrm{K}$.

For the variation of the enthalpy and Prandtl number with temperature, the NBS perfect gas tables are used. The specific heat $c_{p}$ is computed from the definition of Prandtl number using the calculated values of $\mu$ and $k$ and the tabulated values of $P_{r}$.

\section{REFERENCES}

1. Klebanoff, P. S., Tidstrom. K. D., and Sargent, L. M., The Three Dimensional Nature of Boundary Layer Instability. J. Fluid Mech., vol. 1, 1962, pp.1-34.

2. Gad-el-Hak, M., Davis, S. H. McMurray, J. T. and Orszag, S. A., On the Stability of the Decelerating Laminar Boundary Layer. J. Fluid Mech., vol. 138, 1984, pp. 297-323. 3. Hama, F. R., Long, J. D. and Hegarty, J. C., On Transition from Laminar to Turbulent Flow. J. Appl. Phys., vol. 27, 1957, pp. 388-394.

4. Kovasznay, L. S. G., Komoda, H. and Vasudeva, B. R., Detailed Flow Field in Transition. Proc. Heat Transfer Fluid Mech. Inst., 1962, pp. 1-26, Stanford, California, 
Stanford University Press.

5. Kachanov, Yu. S. and Levchenco, V. Ya., The Resonant Interaction of Disturbances at Laminar-Turbulent Transition in a Boundary Layer. J. Fluid Mech., vol. 138, 1984, pp. 209-247.

6. Kachnov, Yu. S., Kosolov, V. V. and Levchenco, V. Ya., Nonlinear Development of a Wave in a Boundary Layer. Fluid Dynamics, vol. 3, 1977, pp. 383-390.

7. Saric, W. S. and Thomas, A. S. W., Experiments on the Subharmonic Route to Transition. Turbulent and Chaotic Phenomena in Fluids, ed. T. Tatsumi, NorthHoland, 1984.

8. Saric, W. S., Kozlov, V. V. and Levchenco, V. Ya., Forced and Unforced Subharmonic Resonance in Boundary Layer Transition. AIAA Paper 84-0007, 1984.

9. Nishioka, M., Asia, M. and Iida, S., An Experimental Investigation of Secondary Instability in Lam inar-Turbulent Transition. ed. R. Eppler and H. Fasel, 1980, pp. $37-$ 46, Berlin, Springer-Verlag.

10. Craik, A. D. D., Nonlinear Resonant Instability in Boundary Layers. J. Fluid Mech., vol. 50, 1971, pp. 393-413.

11. Maseev, L. M., Occurrance of Three-Dimensional Perturbations in a Boundary Layer. Fluid Dynamics, vol. 3, 1968, pp.23-24.

12. Orszag, S. A. and Patera, A. T., Secondary Instability of Wall-Bounded Shear Layers. J. Fluid Mech., vol. 128, 1983, pp. 347-385.

13. Herbert, Th., Secondary Instability of Plane Channel Flow to Subharmonic ThreeDimensional Disturbances. Phys. Fluids, vol. 26, 1983, pp.871-874.

14. Herbert, Th., Three-Dimensional Phenomenon in the Transional Flat Plate Boundary Layer. AIAA Paper 85-0489, 1985.

15. Murdock, J. W., A Numerical Study of Nonlinear Effects on Boundary-Layer Stability. AIAA Paper 77-127, 1977. 
16. Wray, A. A. and Hussaini, M. Y., Numerical Experiments in Boundary Layer Stability. AIAA Paper 80-0275, 1980.

17. Orszag, S. A. and Kells, L. C., Transition to Turbulence in Plane Poiseulle Flow and Plane Couette Flow. J. Fluid Mech., vol. 96, 1980, pp. 159-206.

18. Kleiser, L., Spectral Simulations of Laminar-Turbulent Transition in Plane Poiseuille Flow and Comparison with Experiments. In Proc. Int. Conf. Numer. Meth. Fluid Dyn., 8th,Aachen, Germany. Lecture notes in physics, ed. E. Krause, vol. 170, pp. 280-287, Berlin, Springer-Verlag.

19. Fasel, H. F., Rist, U., and Konzelmann, U., Numerical Investigation of the ThreeDimensional Development in Boundary-Layer Transition. AIAA Paper 87-1203, 1987.

20. Spalart, P. R. and Yang, K. S., Numerical Study of Ribbon-Induced Transition in Blasius Flow. J. Fluid Mech., vol. 178, 1987, pp. 345-365.

21. Herbert, Th., Secondary Instability of Boundary Layers, Ann. Rev. Fluid Mech., vol. 20,1988 , pp. $487-526$.

22. Bayly, B. J., Orszag, S. A. and Herbert, Th., Instability Mechanisms in Shear-Flow Transition. Ann. Rev. Fluid Mech., vol. 20, 1988, pp. 359-391.

23. Singer, B., Reed, H. L. and Ferziger, J. H., Investigation of the Effect of Initial Disturbances on Plane Channel Transition. AIAA Paper 86-0433, 1986.

24. Singer, B., Ferziger, J. H. and Reed, H. L., Effect of Streamwise Vortices on Transition in Plane-Channel Flow. AIAA Paper 87-0048, 1987.

25. El-Hady, N. M., Evolution of Resonant Wave Triads in Three-Dimensional Boundary Layer. Phys. Fluids A, vol.1, 1989, pp. 549-563.

26. Mack, L. M., Review of Linear Compressible Stability Theory. In Stability for Time Dependent and Spatially Varying Flows, ed. D. L. Dwoyer and M. Y. Hussaini, 1985, pp. 164-187, Springer-Verlag, New York. 
27. Laufer, J. and Vrebalovitch, T., Stability and Transition of a Supersonic Laminar Boundary Layer on an Insulated Flat Plate. J. Fluid Mech., vol. 9, 1960, pp. 257-299.

28. Kendall, J. M., Jr., Supersonic Boundary Layer Stability Experiments. Boundary Layer Transition Study Group Meeting, Vol. II-Session on Boundary-Layer Stability, W. D. McCauley, ed., BSD-TR-67-213, Yol. II, U.S. Air Force, Aug. 1967, pp. 10-1 10-8. (Available from DTIC as AD 820364 ).

29. Kendall, J. M., Wind Tunnel Experiments Relating to Supersonic and Hypersonic Boundary-Layer Transition. AIAA J., vol. 13, 1975, pp.290-299.

30. Demetriades, A., An Experiment on the Stability of Hypersonic Boundary Layers. J. Fluid Mech., vol. 7, 1960, pp. 385-396.

31. Demetriades, A., Hypersonic Viscous Flow Over a Slender Cone: Part III: Laminar Instability and Transition. AIAA Paper 74-535, 1974.

32. Stetson, K. F., Thompson, E. R., Donaidson, J. C. and Siler, L. G., Laminar Boundary-Layer Stability Experiments on a Cone at Mach 8. Part 1: Sharp Cone. AIAA Paper 83-1761, 1983.

33. Erlebacher, G. and Hussaini, M. Y., Stability and Transition in Supersonic Boundary Layer. AIAA Paper 87-1416, 1987.

34. El-Hady, N. M., Secondary Three-Dimensional Instability in Compressible Boundary Layers. Transonic Symposium: Theory, Application, and Experiment, NASA CP-3020, vol. I, part 2, 1988, pp. 691-704.

35. Mack, L. M., Boundary-Layer Stability Theory, NASA CR-131501, 1969.

36. El-Hady, N. M. and Nayfeh, A. H., Nonparallel Stability of Compressible Boundary-Layer Flows. AIAA Paper 80-0277, 1980.

37. El-Hady. N. M., On the Effect of Boundary-Layer Growth on the Stability of Compressible Flows. NASA CR -3474, 1981. 
38. Scott, M. R. and Watts, H. A., Computational Solution of Linear two-Point Boundary Value Problems Via Orthonormalization. SIAM J. Numer. Anal., vol. 14, 1977, pp.40-70.

39. Herbert, Th., Subharmonic Three-Dimensional Disturbances in Unstable Plane Shear Flows. AIAA Paper 83-1759, 1983. 40. Nayfeh, A., H., Three-Dimensional Spatial Secondary Instability in Boundary-Layer Flows. AIA A Paper 85-1697, 1985. 


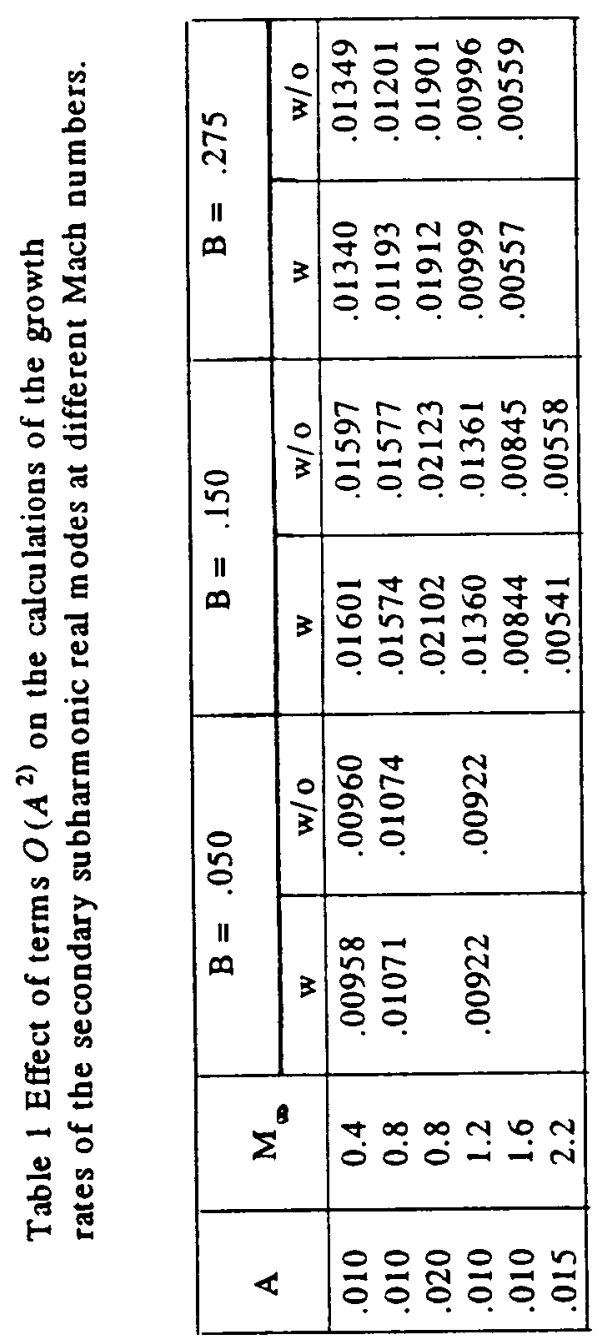



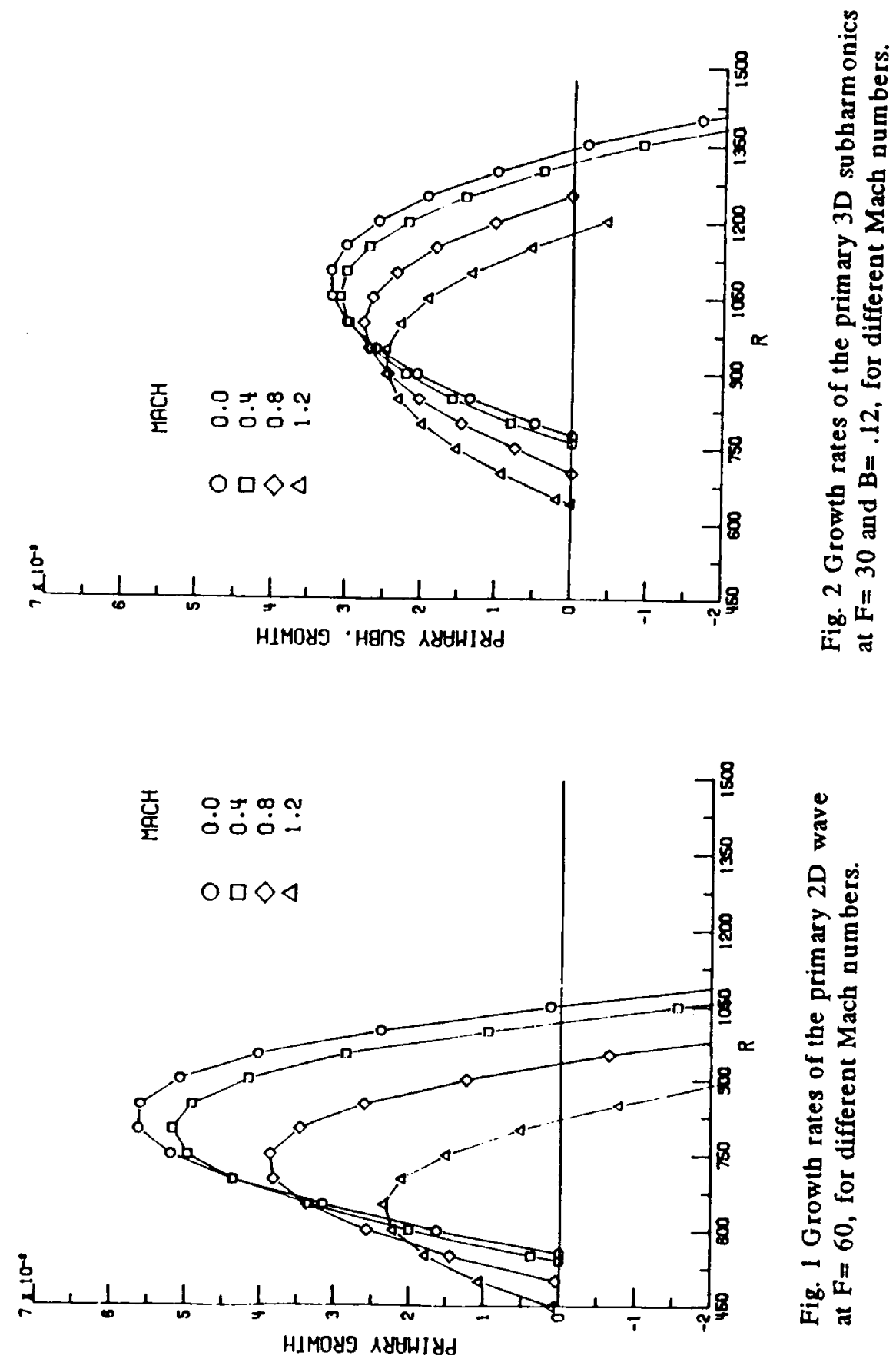


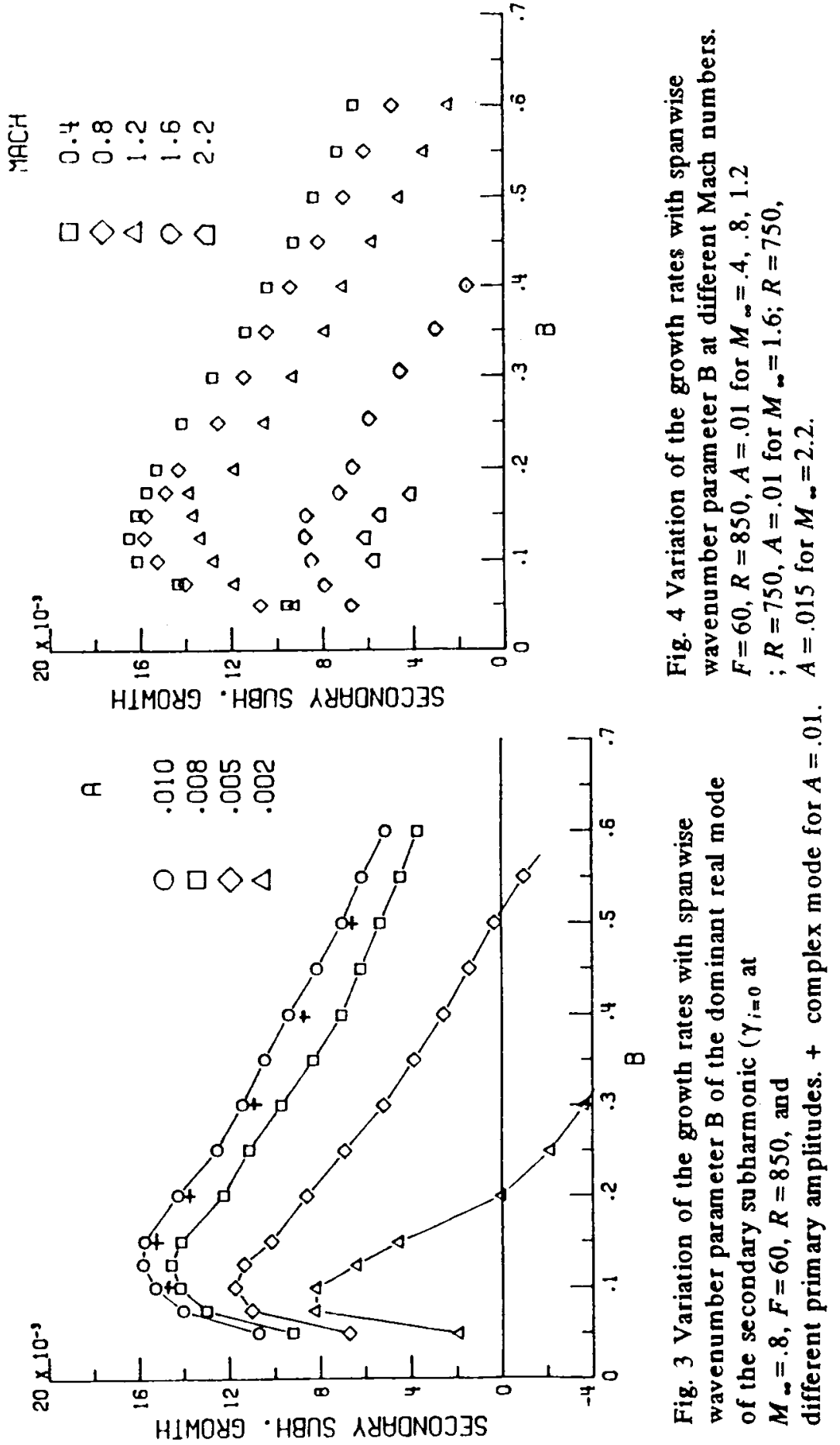




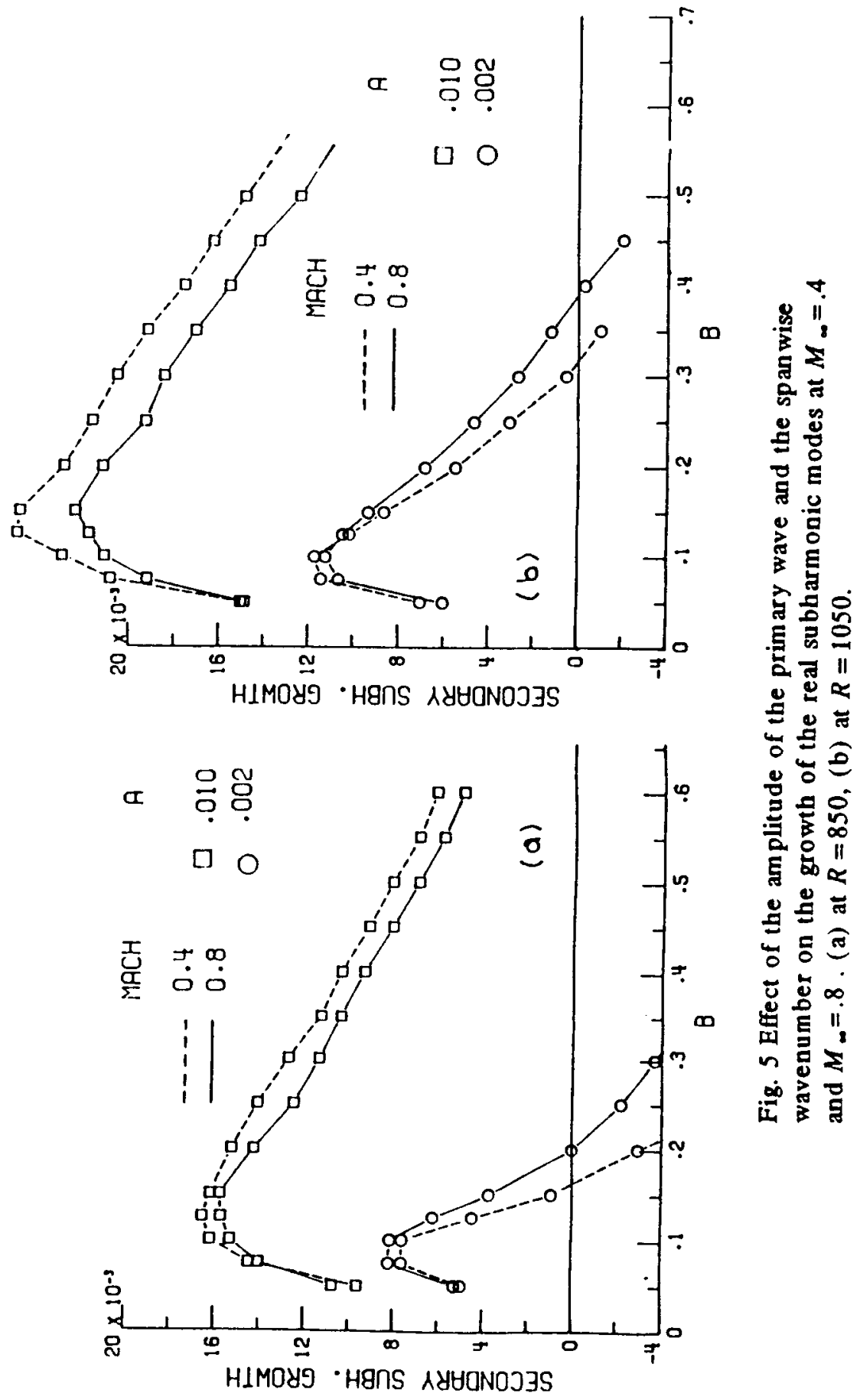




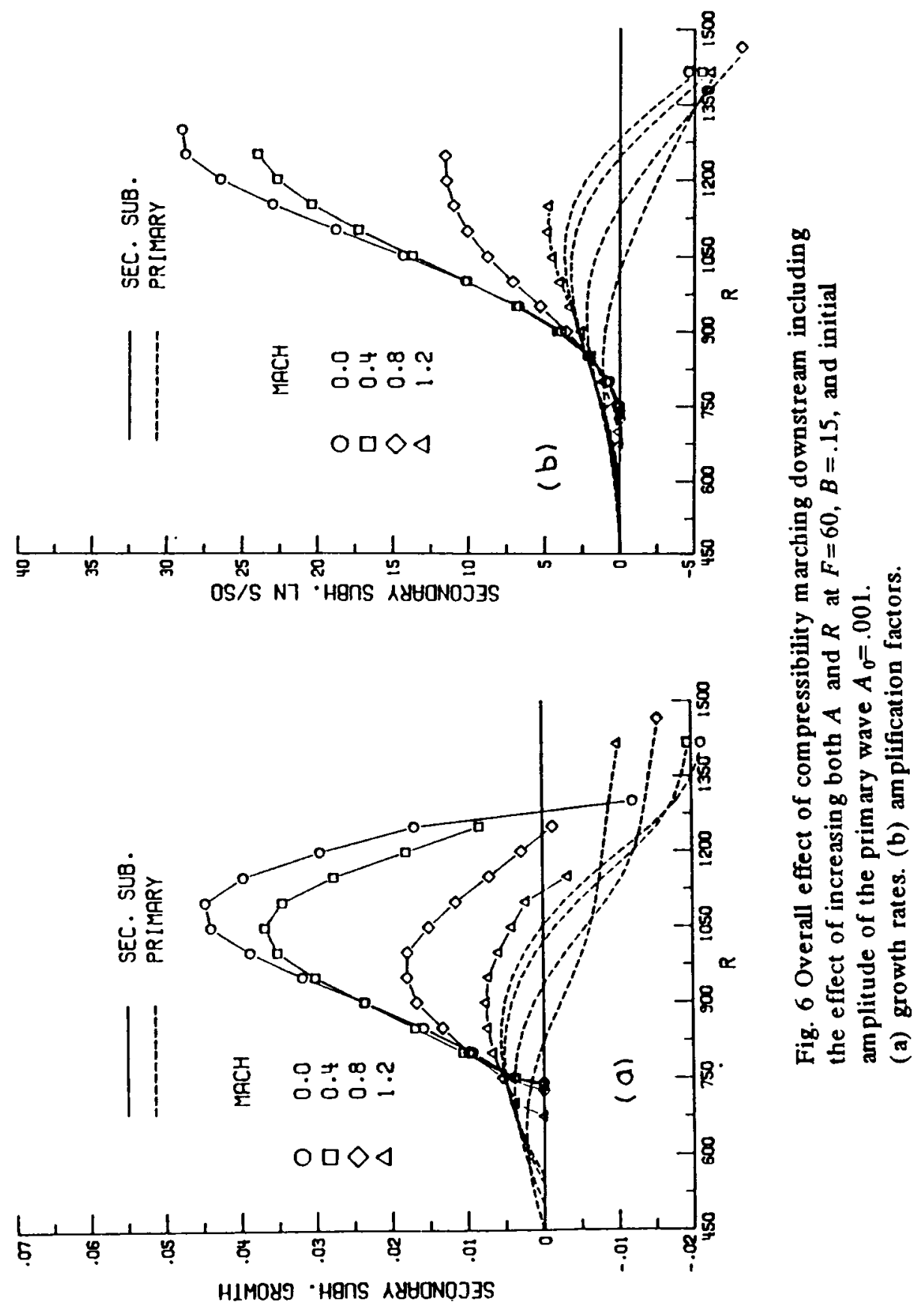



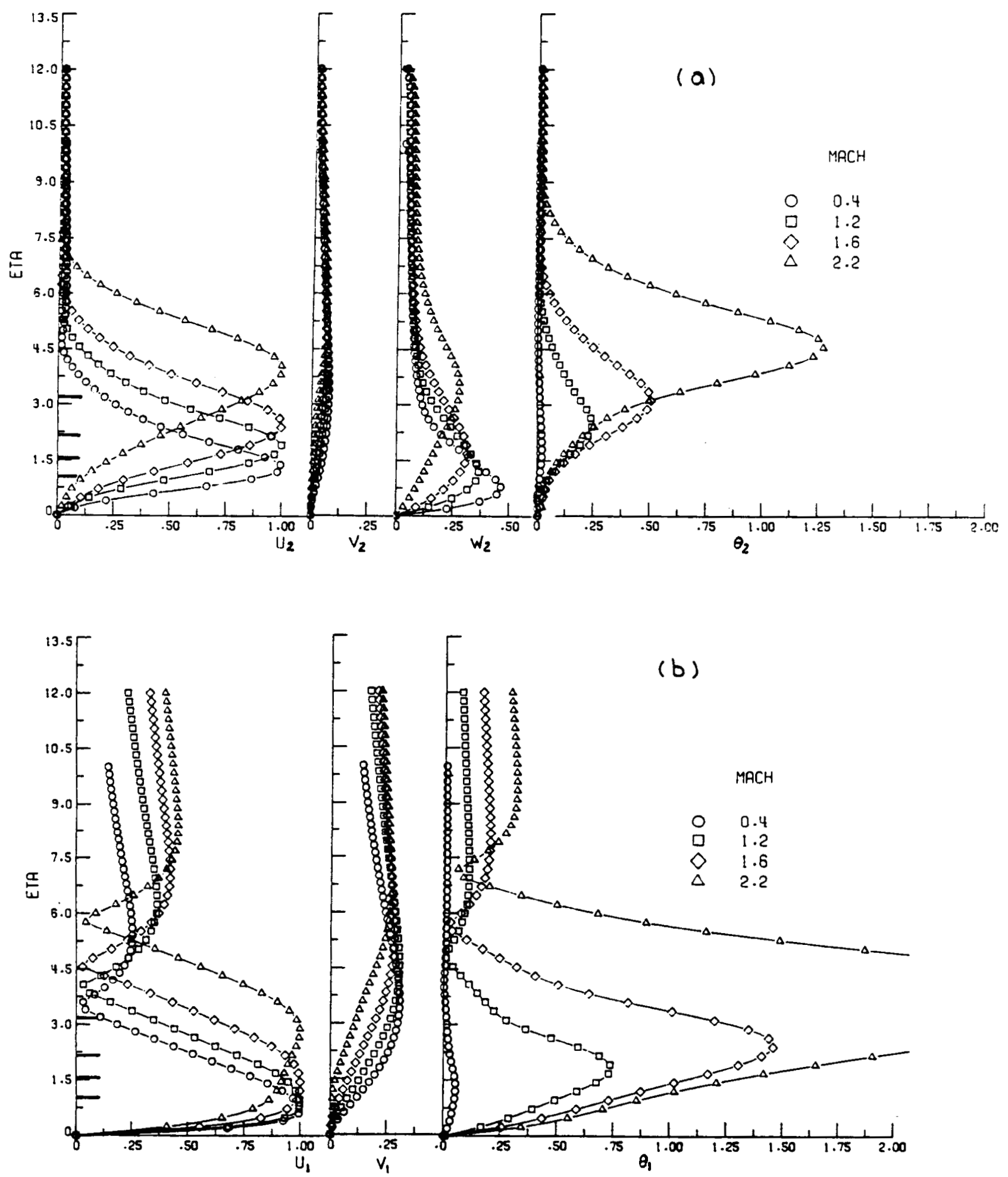

Fig. 7 Variation across the boundary layer of the eigenfunctions at different Mach numbers and the conditions of fig.4. (a) Secondary subharm onic disturbance. (b) Prim ary wave. 


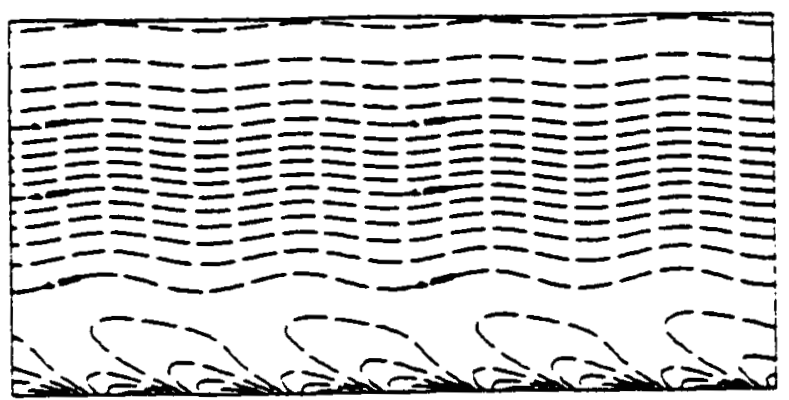

(a)

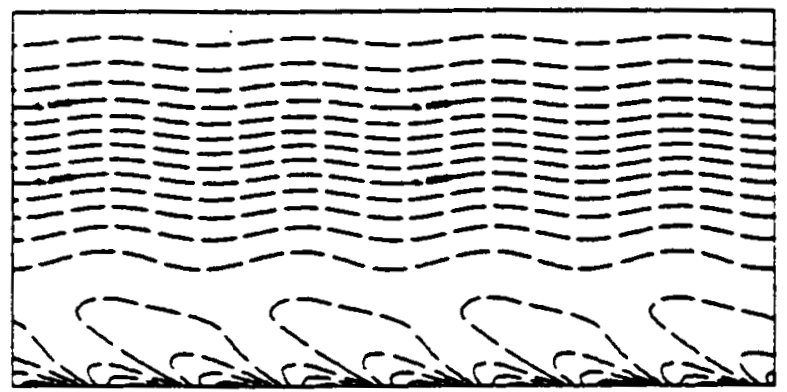

(b)

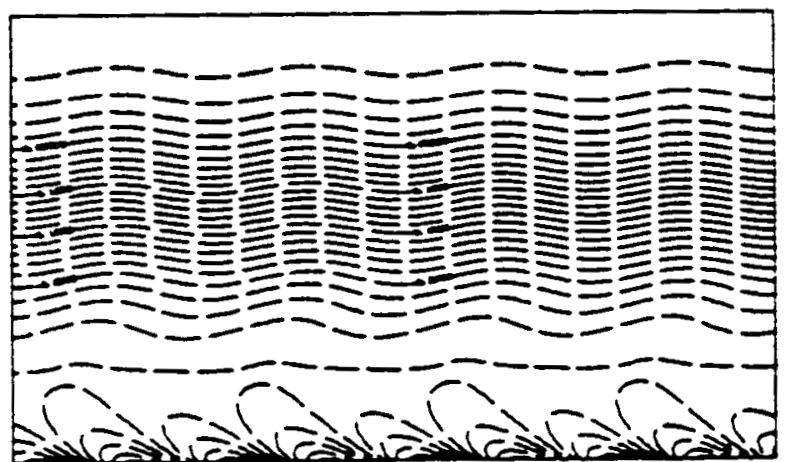

(c)

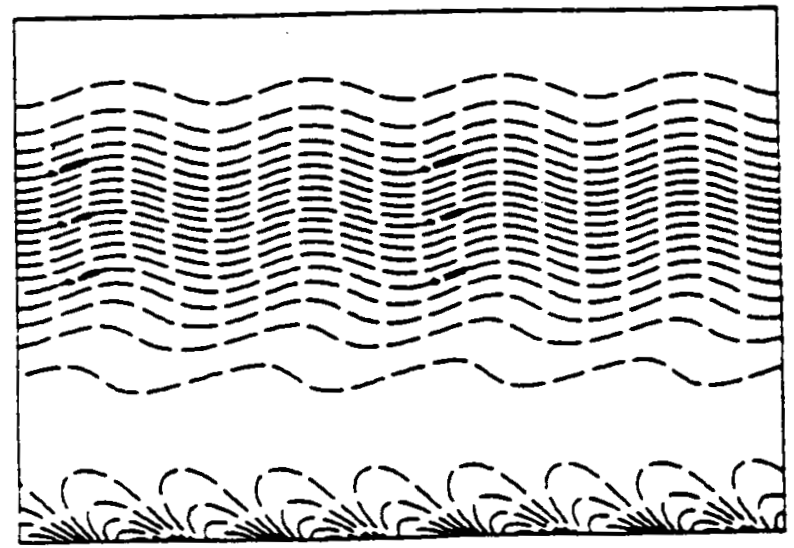

(d)

Fig. 8 Initial 2D vorticity contours of the basic flow at different Mach numbers. (a) $M_{-}=.4$, $R=850, A=.01$; (b) $M_{-}=.8, R=850, A=.01$; (c) $M_{-}=1.6, R=750, A=.015$; (d) $M_{-}=2.2$, $R=750, A=.015$. 

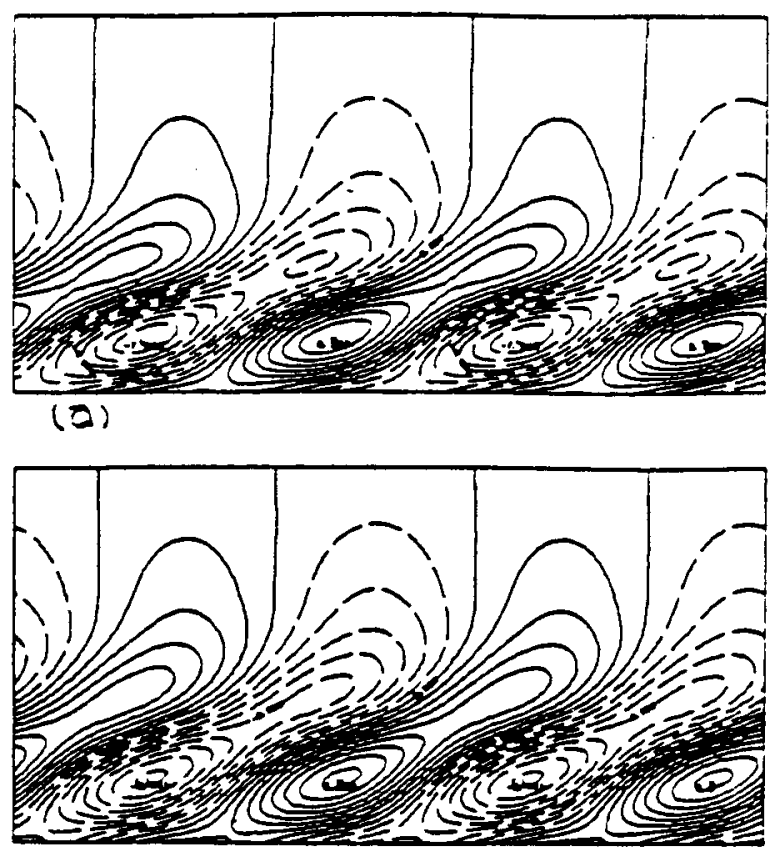

(b)

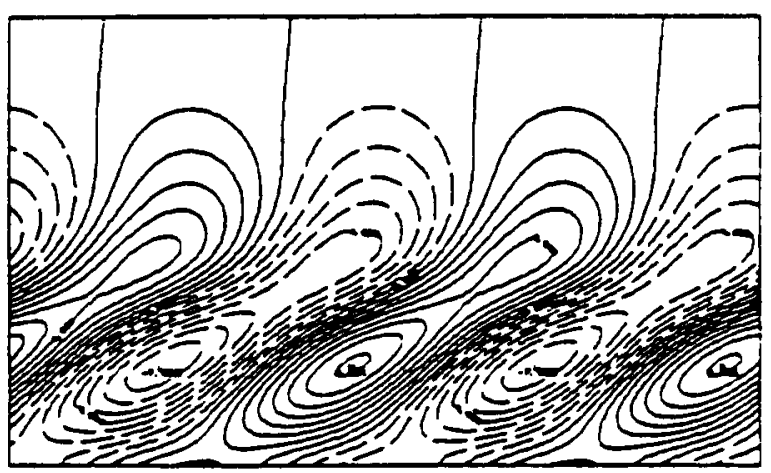

(C)

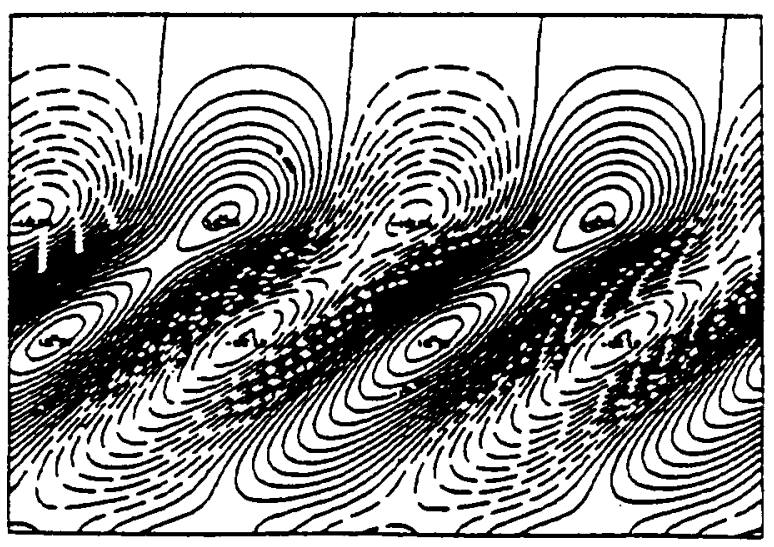

(d)

Fig. 9 Span wise vorticity contours of the 3D fiow in the $x-y$ plane at $z=0$, at different Mach numbers. Same conditions of fig. 8 . 


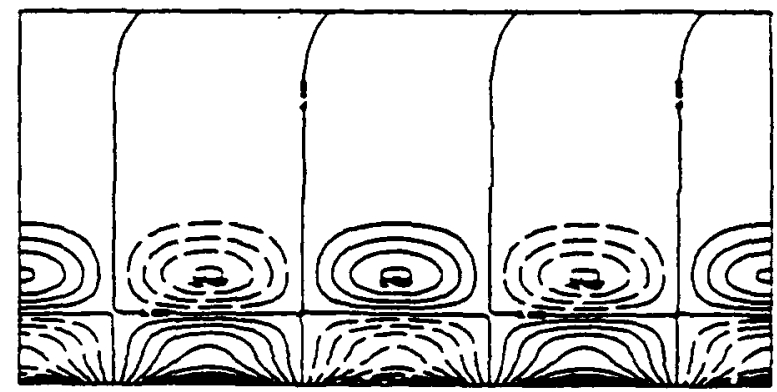

(a)

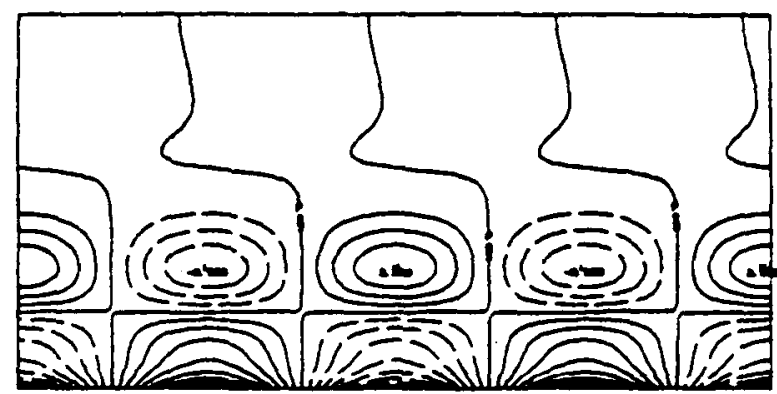

(b)

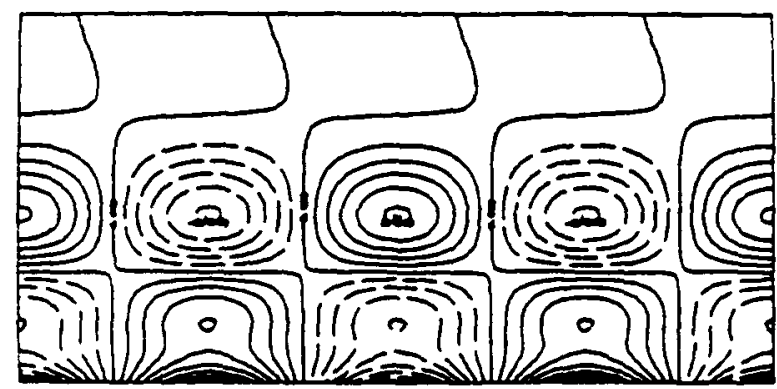

(c)

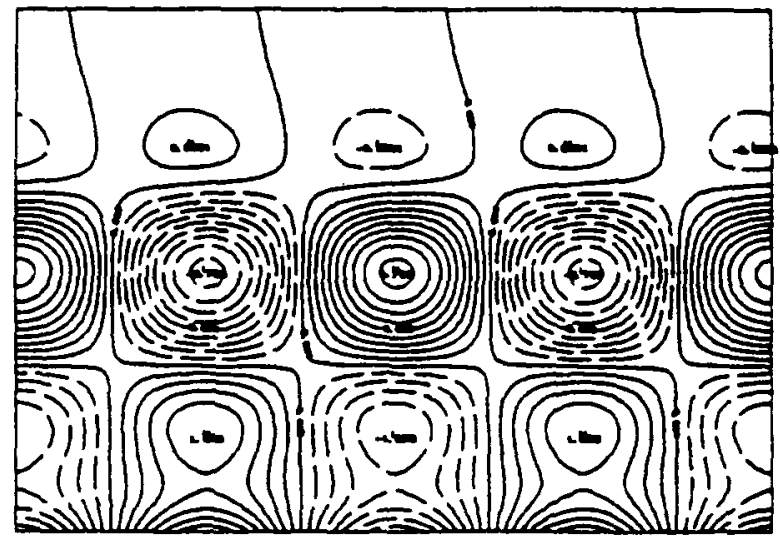

(d)

Fig. 10 Stream wise vorticity contours of the 30 flow in the $z-y$ plane at $x=0$ at different Mach numbers. Same conditions of fig. 8 . 


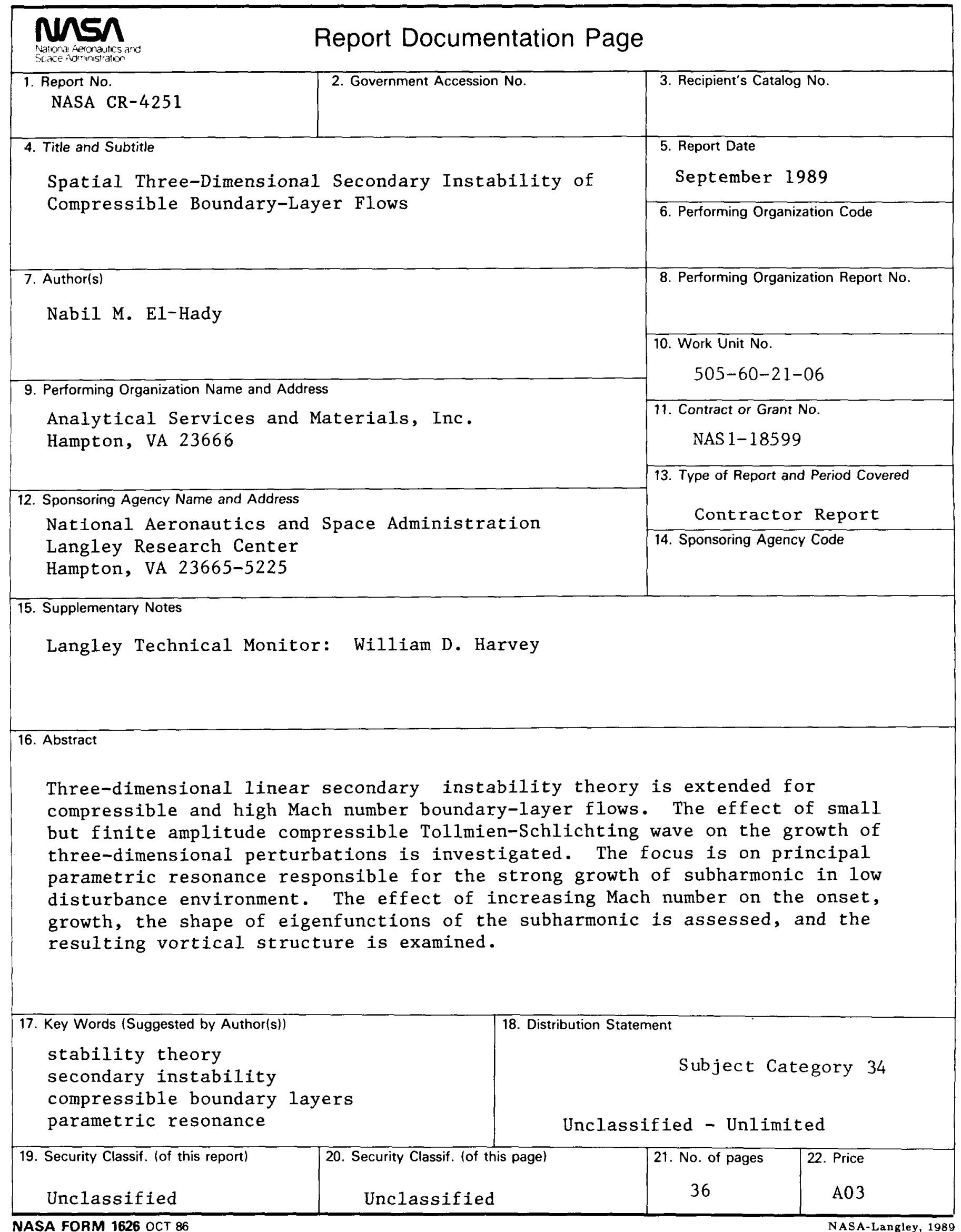

For sale by the National Technical Information Service, Springfield, Virginia 22161-2171 